\title{
Improved greedy routing protocol for VANET
}

\author{
Ambica \\ Department of computer science \\ BGIET ,Sangrur \\ Punjab,India
}

\author{
Avinash Jethi \\ Department of computer science \\ BGIET ,Sangrur \\ Punjab,India
}

\begin{abstract}
VANET (vehicular ad-hoc network) is a classification of MANET in which vehicles act as mobile node and provides a different approach to Intelligent transport System (ITS). VANET is an emerging area for Intelligent Transportation System (ITS) which can result in increased traffic safety, collision warning through exchange of messages through wireless media. Efficient routing protocols are required for efficient communication among vehicles. In the given paper, we surveyed various VANETs protocols and along with its advantages and disadvantages. We compare our proposed protocols via NS-2 based simulations and show the performance of different protocols.
\end{abstract}

keywords: VANET, routing protocols, GPRS,GSPR-MV,V2V

\section{INTRODUCTION}

Vehicular ad hoc network (VANET) is becoming a promising area in the field of wireless network. VANET is a subset of MANET in which vehicles act as mobile node that provides communications among nearby vehicles, and between vehicles and nearby road side equipments through wireless medium. The U.S. Federal Communications Commission (FCC) has recently allocated the 5.85- $5.925 \mathrm{GHz}$ portion of the spectrum to intervehicle communication (IVC) and vehicle-to-roadside communication (VRC) under dedicated short-range communications (DSRC). The vehicles and the associated infrastructure (all nodes) are equipped with wireless devices to gather data, and the data is processed to determine present traffic conditions and disseminate it over longer distances and can use it to alter its decision. For example they can change their route based on the information received in case of any congestion or collision warning. Regular enhancement in infrastructure of VANETs can result in increased safety and comfort of passenger and driver. VANET approach is scalable and has low maintenance costs. Government is taking much interesting in VANET because of safety it provides. Many projects such as COMCAR [15] , CarTALK [16], CarNet [17]. Characteristics of VANETs are [14] -

- $\quad$ Frequent Disconnected Network

- Dynamic topology.

- Battery Power and Storage Capacity

- Communication Environment
- Interaction with onboard sensors

VANET provide communication with vehicles in three modes

- Inter-vehicle communication

- Vehicle-to-roadside communication, and

- Routing-based communication

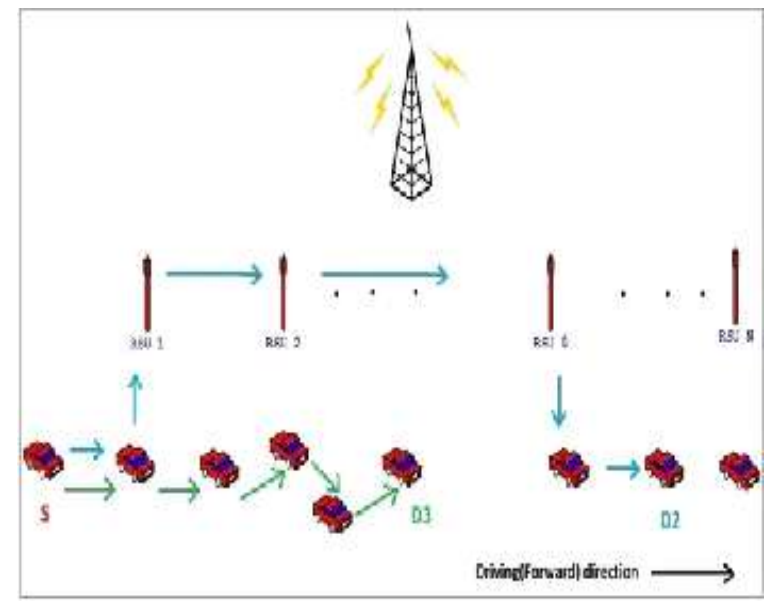

VANET routing protocols are usually designed for urban environments where vehicles are equipped with wireless connectivity and a GPS device. Topology is dynamic and the routing protocol must be fast enough to find a route in order to have a good response time. Based on the location of vehicle list of its neighbors is generated which are then used for discovery of route from source to destination. The consumption of battery in vehicles is very fast so in order to improve performance and energy conservation in fast moving vehicles light weight routing 
protocols need to be defined in Physical and Data link layer. For discovering the route ad hoc network use two different types of protocols which are classified as Proactive and Reactive [18] protocols. Proactive protocols maintain the routing information from each node to every node at all the time and that is up-to-date. Reactive protocols, also called "on demand" node initiates a route discovery process only when a route to destination is required.

In this paper, Section II describes the VANET architecture. Section III presents a survey of these protocols experimented on to VANET and their advantages and disadvantages.

\section{VANET NETWORK ARCHITECTURE}

Architecture of vanet network can be classified into 3 categories: cellular WLAN, pure ad hoc and hybrid.

\subsection{Cellular /WLAN}

Fixed cellular gateways and WLAN/WiMAX access points are used at traffic intersection to connect to internet and gather information. VANET combines both cellular and WLAN network so that WLAN is used where an internet connection is available. But it adds to cost because of fixed gateways and other hardware devices. Figure 1 shows cellular/WLAN architecture.

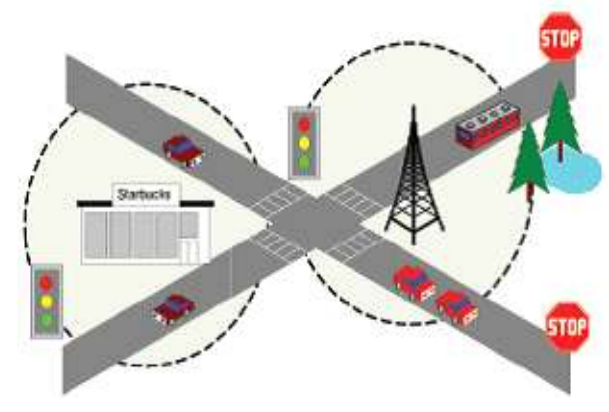

Figure 1 Cellular/WLAN Network Architecture

\subsection{Ad hoc}

To overcome the shortcoming of cellular/WLAN network vehicles can form an ad hoc network among themselves. Figure 2 shows an ad hoc network. It helps in vehicle to vehicle communications and achieves certain goals, such as blind crossing.

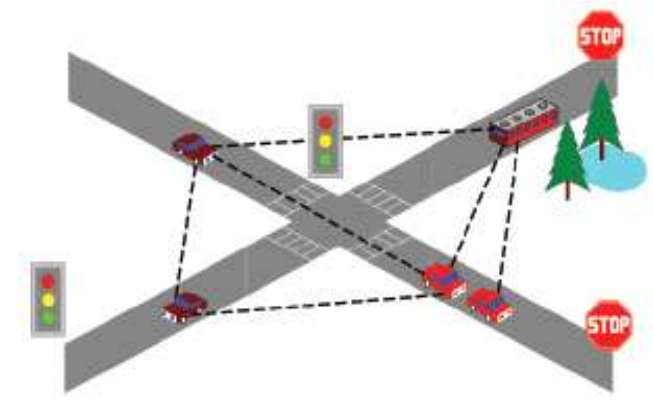

Figure 2 Ad hoc Network

\subsection{Hybrid}

Hybrid architecture is a combination of infrastructure and ad hoc network. It uses vehicles with both WLAN and cellular capabilities as gateways and mobile network routers so that vehicles with only WLAN capability can communicate with them through multi-hop links to remain connected to the world. The hybrid architecture can provide better coverage, but also causes new problems, such as the seamless transition of the communication among different wireless systems.

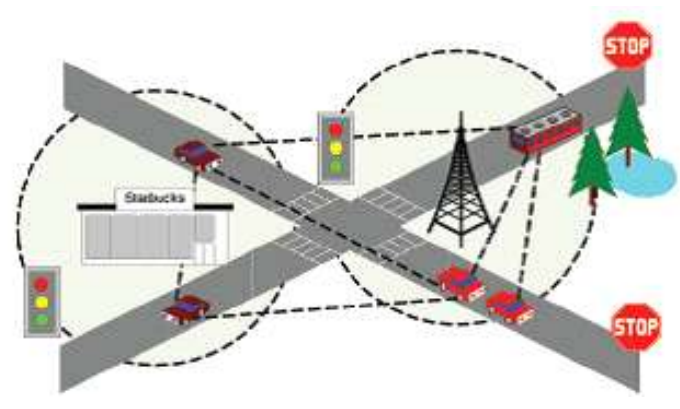

Figure 3 Hybrid Architecture

\section{ROUTING PROTOCOLS IN VANET}

Routing protocols for VANET are designed for urban environment where vehicles are equipped with GPS devices and wireless devices for continuous tracking of vehicles. The goal of routing protocols is to select an optimal path with minimum cost. Due to dynamic behavior of VANET network its topology keep on changing. So as to assure the messages are delivered from source to destination time evolving networks should be applied.

Vehicular ad-hoc routing is classified as Unicast, Multicast and Broadcast routing. Unicast routing protocols is useful in vehicle to vehicle communication. Multicast routing is used to disseminate the message to specified area and effective in conditions like traffic jam and accidents. Broadcast routing is 
useful to communicate with all the nodes in its transmission range and helpful in conditions like bad road and whether conditions.

In this paper, we focus mainly on unicast routing protocols. The routing protocols for VANET are classified in following categories

\subsection{Topology Based Routing}

This routing protocol uses topology that exists in the network to forward packets. They are Proactive and Reactive routing protocols.

\subsubsection{Proactive Routing Protocol}

These kind of routing protocol stores routing information like next forwarding hop. Periodic exchange of topology of network is exchanged among nodes to maintain the paths between any pair of nodes even though some of paths are never used.. A table is then constructed within a node such that each entry in the table indicates the next hop node toward a certain destination. They do not have initial route discovery delay but consumes lot of bandwidth for periodic updates of topology. Various types of Proactive Routing protocols are DSDV, OLSR, FSR, CGSR, WRP.

\section{Advantage}

- In these types of protocols, there is no route discovery since the destination route is stored in background.

- They have the best end to-end delay and packets delivery fraction but at the cost of higher routing load.[ Comparative Study of Reactive and Proactive Routing]

\section{Disadvantage}

- It provides low latency for real time applications.

\subsubsection{Reactive Routing Protocol}

It is an on demand routing protocol which opens a route only when a node wants to communicate with another node. It maintains the information of only those nodes that are currently being used by network. Thus, reducing burden on network. These types of protocols have a route discovery phase where query packets are flooded into the network in search of a path. The phase completes when a route is found. Reactive routing protocols are applicable to the large size of the mobile ad-hoc networks which are highly mobility and frequent topology changes. AODV, TORA, PGB, DSR are some of reactive protocols.

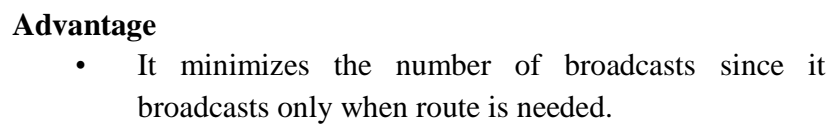

\author{
Disadvantage \\ - Excessive flooding can disrupt the network. \\ - Route finding latency is high.
}

\subsection{Position Based Routing/ Geographic Routing}

It is a routing technique in which a node makes its routing decision with the help of information received from GPS device. It sends packet from source to destination based on the geographic position of vehicle instead of using network address. It does not maintain any routing table or exchange link state information with another node in network. In this each node knows its position and its neighbor's position and uses that information to make routing decision. When the source need to send a packet, it stores the destination address in the packet header which will help in forwarding the packet to the destination without needs to route discovery, route maintenance, or even awareness of the network topology. It can be classified as Position based greedy V2V protocols, Delay Tolerant Protocols.

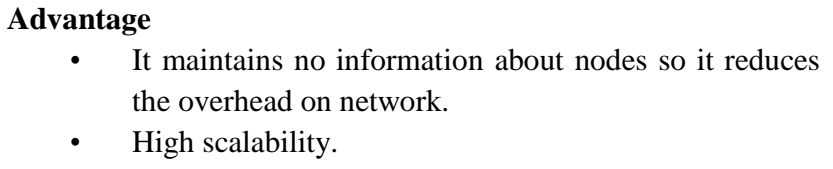

- It maintains no information about nodes so it reduces the overhead on network.

- High scalability.

\section{Disadvantage}

- Dependent on GPS service.

\subsubsection{Position Based Greedy V2V Protocols}

GPSR makes the greedy forwarding decision based only on the information about immediate neighbors of a node .when packet reaches a region where greedy forwarding is impossible, the algorithm recovers by routing around the perimeter of the region. There some problem occur with protocol. To solve this problem in this paper we used GSPR-MV protocol.

\section{GSPR-MV}

This is called greedy simplified perimeter routing with moving vector. It includes:

- Improved GPSR-MV

- $\quad$ Simplified of perimeter forwarding

In improved GPSR-MV the mobile nodes makes packet forwarding decision directly according to itself, the neighboring nodes and location information of the destination node. There are two kinds of: Greedy and perimeter mode.

In Simplified perimeter forwarding, in the GPSR protocol the nodes listen to transmitted data packets and the no. of experienced hops from the source node to the current node after that the data packet transmission the redundant paths can be avoided and this makes the routes more concise and robust. 


\subsubsection{Delay Tolerant Protocols}

Due to high dynamic topology of VANET frequent disconnection among vehicles is common. It uses carry and forward strategy. It allows nodes to store information when there is no contact between other nodes and it can carry on with that information until it meets with other nodes.

\subsection{Cluster based Routing Protocol}

This type of routing protocol divides the network into cluster with similar characteristics like topology, speed, velocity etc. Cluster of vehicles is formed and each cluster has a cluster head which is responsible for inter and intra cluster communications. For intra cluster communication, a virtual link is created between nodes for communication and for inter cluster communication cluster communicates through cluster head. Various Clusters based routing protocols are $\mathrm{HCB}, \mathrm{CBR}$, COIN, TIBCRPH, CBLR, CBDRP etc.

\section{Advantage}

- It provides good scalability for large networks.

\section{Disadvantages}

- Due to dynamic topology of VANET the delay and overhead of maintaining these clusters is large.

\subsection{Broadcast Based Routing}

The simplest way to implement broadcasting is flooding. It is a flooding based routing protocol in which the message is flooded to all nodes in network except form where the message came. It is used by VANET for sharing information like its position, traffic, weather and emergency, road conditions among vehicles to maximum nodes possible. Flooding guarantees that the message will eventually reach all the nodes i.e. vehicles in the network. But in a large network, it causes exponential increase in message transmission resulting in collision so it increases the overhead and decreases the performance of network. Broadcast routing protocols are BROADCOMM, UMB, V-TRADE, DVCAST, EAEP, SRB, PBSM, PGB, DECA, POCA etc.

\section{Advantage}

- Information reaches every node in much less time.

\section{Disadvantage}

- Many nodes receive duplicate packets.

- $\quad$ Reduces the network bandwidth.

\subsection{Geocast Based Routing Protocol}

Geocast routing is a location-based multicast routing. It works by delivering the packet from source to destination within a specified geographical area. The selected area for transmission is called Zone of Relevance or ZOR. The main idea is that sender node need not to packet to nodes beyond the ZOR. Direct flooding strategy is used to reduce the amount of overhead and network congestion when packets are flooded. The various Geocast routing protocols are IVG, DG-CASTOR, DRG.

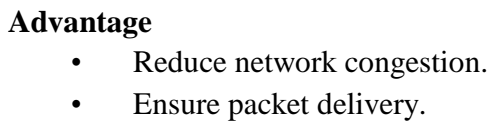

\section{Disadvantage}

- It requires more latency and control overhead to create routes

\section{Conclusion}

In this paper, architecture of VANET and advantages and disadvantages of VANET Routing protocols are discussed. There are number of schemes for handling routing and information dissemination but there are few which deal with safety requirements due to overhead in discovering and maintaining routes. To evaluate the performance of various protocols in VANET can be evaluated based on various performance parameters. Routing vehicle safety communications remain a challenging task.

\section{REFERENCES}

[1] "Survey of Routing Protocols in Vehicular Ad Hoc Networks," Kevin C. Lee, Uichin Lee, Mario Gerla, Advances in Vehicular Ad-Hoc Networks: Developments and Challenges, IGI Global, Oct, 2009.

[2] "Evaluation of Proactive, Reactive and Hybrid Ad hoc Routing Protocol for various Battery models in VANET using Qualnet" Manish Sharma, Gurpadam Singh, International Journal of Smart Sensors and Ad Hoc Networks (IJSSAN) ISSN No. 2248-9738 (Print) Volume-1, Issue-2, 2011.

[3] "A Comparative Study of Routing Protocol in Vehicular Ad Hoc Network" Pooja Duddalwar, Atul Deshmukh, S. S. Dorle, International Journal of Emerging Technology and Advanced Engineering ISSN 2250-2459, Volume 2, Issue 3, March 2012.

[4] "A Comparative Study of Various Routing Protocols in VANET" Rakesh Kumar, Mayank Dave International Journal of Computer Science Issues, Vol. 8, Issue 4, No 1, July 2011

[5] "A Survey on Routing Mechanism and Techniques in Vehicle to Vehicle Communication (VANET)" Mr. Yugal Kumar, Mr. Pradeep Kumar and Mr. Akash Kadian, International Journal of 
Computer Science \& Engineering Survey (IJCSES) Vol.2, No.1, Feb 2011.

[6] "Comparative and Behavioral Study of Various Routing Protocols in VANET" Udit Agarwal, Monika Saxena, International Journal of Advanced Research in Computer Science and Software Engineering Volume 3, Issue 10, October 2013.

[7] "A study on the feasibility of mobile gateways for vehicular ad-hoc networks," V. Namboodiri, M. Agarwal, and L. Gao, in proceedings of the First International Workshop on Vehicular Ad Hoc Networks, pp. 66-75, 2004.

[8] "Performance analysis of AODV \&GPSR routing protocol in VANET" Neeraj Sharma, Jawahar Thakur International Journal of Computer Science \& Engineering Technology (IJCSET) Vol. 4 No. 02 Feb 2013.

[9] "PERFORMANCE EVALUATION OF REALISTIC VANET USING TRAFFIC LIGHT SCENARIO” Nidhi ,D.K Lobiyal, International Journal of Wireless \& Mobile Networks (IJWMN) Vol. 4, No. 1, February 2012.

[10] "PERFORMANCE COMPARISON OF POSITIONBASED ROUTING PROTOCOLS IN VEHICLE-TOVEHICLE (V2V) COMMUNICATION" RAM SHRINGAR RAW, SANJOY DAS, International Journal of Engineering Science and Technology (IJEST) Vol. 3 No. 1 Jan 2011.
[11] "A Survey on Routing Protocols and its Issues in VANET" Jagadeesh Kakarla, S Siva Sathya, B Govinda Laxmi, Ramesh Babu B International Journal of Computer Applications (0975 8887)

Volume 28- No.4, August 2011.

[12] "Survey on security challenges in VANET" Anup Dhamgaye, Nekita Chavhan, International Journal of Computer Science and Network, Vol 2, Issue 1, 2013.

[13] "Survey over VANET Routing Protocols for Vehicle to Vehicle Communication" Bijan Paul, Mohammed J. Islam IOSR Journal of Computer Engineering (IOSRJCE) Volume 7, Issue 5 (Nov-Dec. 2012), PP 01-09.

[14] "VANET Routing Protocols: Pros and Cons" Bijan Paul, Md. Ibrahim, Md. Abu Naser Bikas, International Journal of Computer Applications (0975 - 8887) Volume 20- No.3, April 2011.

[15] Ericson, "Communication and Mobility by Cellular Advanced Radio", ComCar project, www.comcar.de, 2002.

[16] Reichardt D., Miglietta M., Moretti L., Morsink P., and Schulz W.,"CarTALK 2000 - safe and comfortable driving based upon inter-vehicle-communication," in Proc. IEEE IV'02.

[17] "GSPR-MV: a routing protocol based on motion vector for Vanet”Hongyu Tu,Lei Peng, Huiyun li,Falin liu,in IEEE 2014. 